incidence of adenocarcinomas may be influenced by the staining methods used. Some standardization of technique is desirable and the alcian blue-PAS combination appears to be the most satisfactory.

\section{The Nuclear Channel System of the Human Endometrial Glandular Cell}

I. A. R. MORE AND E. M. ARMSTRONG (Western Infirmary, Glasgow, introduced by R. N. M. MACSWEEN) Endometrial curettings from 56 healthy women at all stages of the menstrual cycle were surveyed for the occurrence of nucleolar channel systems. Typical nucleolar specializations were noted in 14 of 26 women biopsied between day 13 and day 26 of the menstrual cycle and were observed most commonly and in greatest numbers between day 17 and day 20 .

The nucleolar channel system occurs as an ordered, angular mass of interdigitating membrane-bound tubules of 60-100 $\mathrm{nm}$ diameter embedded in a dense granular matrix surrounding a core of lightly granular material. It arises in association with an invagination of both inner and outer nuclear membrane.

Towards the end of the cycle the nucleolar channel system appears more commonly as a dense disordered mass of tubules lacking a central core, often occurring as a protrusion of the nucleus. Although such masses have not yet been positively identified free in the cytoplasm, micrographs suggesting extrusion of the channel system and possible incorporation into giant lysosomes are presented.

The function of the structure is discussed. In particular its appearance is linked with the presence of 17 B progestational steroids and it is suggested that the tubular system itself may be a manifestation of a specific hormoneinduced gene derepression. The structure may therefore provide a pathway for the rapid transport of newly formed m RNA molecules into the cytoplasm where they could act as a template for new protein synthesis.

\section{Pyogenic Granuloma of the Urinary Bladder}

C. K. ANDERSON (University of Leeds) Pyogenic granuloma of the urinary bladder is a condition found in a small group of patients presenting with severe lower urinary tract symptoms, usually pain, frequency, and haematuria. Seven cases have been seen in a provincial urological clinic over a period of 12 years.

Clinically, the patients are usually in the fifth and sixth decades, although the conditions may be seen in younger patients. There is a preponderance of females to males ( $5: 2$ in this series). The urine does not contain malignant cells and there is no consistent pattern of urinary tract infection, most patients having a sterile urine on presentation or during the course of the condition. Intravenous pyelography is usually normal, but may show a dilated upper tract $(2 / 7)$ or a filling defect in the bladder (1/7). On cystoscopy the lesion appears red and angry with irregular elevation of the bladder mucosa resembling an infiltrating neoplasm. The lesion usually appears single.

Histologically the lesion is always covered by intact mucosa in the early stages. The epithelium is usually regular. The submucosa contains congeries of vascular spaces with area of vasoformative tissue infiltrated by inflammatory cells, including many pus cells. No organisms are seen in the fixed tissue preparations. Electron microscopy shows no inclusion bodies and no visible virus particles.

The lesion may regress spontaneously leaving an area of scar tissue under the bladder mucosa; in one case progression to leukoplakia occurred. Generally the lesion persists with continuing symptoms unless resected endoscopically or excised by segmental cystectomy. In two patients additional lesions have arisen. Immunological studies have been made on one patient and show an apparent deviation of complement into the lesion.

Fibrin and Complement in Glomerulonephritis

A. M. DAVISON, D. THOMSON, AND MARY K. MACDONALD (University of Edinburgh, Edinburgh) Glomerulonephritis may be induced in experimental animals by immunological means and it is thought that some forms of human glomerulonephritis are due to such mechanisms. Immunological reactions may be associated with activation of the coagulation and complement systems with subsequent deposition of complement and fibrin.

Histologically it is possible to classify glomerulonephritis on the basis of morphological appearance. However it is now recognized that identical histological appearances may be produced by a wide

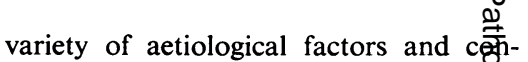
versely a single precipitating factor maay induce differing histological patter $\overrightarrow{f s}$. Electron microscopy is of further vafue in characterizing the ultrastructural features of glomerulonephritis, but iojs not possible to determine the nature ${ }_{0} \mathrm{f}$ deposited material by this meth Immunofluorescence microscopy is of considerable help in elucidating the composition of the material deposited within the glomerulus in a patient with glomerulonephritis. There is a gogod relationship in the site of abnorffal material as observed by immupofluorescence and electron microscos In addition examination of the urine from patients with glomerulonephritis for flie degradation products of fibrin $\varkappa_{d}$ complement reveals a good relationslitp between their excretion and the preseffe of such material within the glomerulus?

It has been possible to demonstrate that the mesangial cell is responsigle for removing material deposited within the glomerular capillary walls, and indeged for the ability to recover from a gigen insult. It may also be the case that the histological pattern is determined once by the ability of the mesangial celcto remove adequately the producte. - of immunological injury than by the of the primary aetiological factor.

\section{Serratia marcescens Infection General Hospital}

W. A. BLACK, L. A. HATCH, P. BINNIE, AD JEAN NEWBERRY (St Joseph's Hospitol, London, Ontario, introduced by H. SISSONS) In recent years increasing attention has been given to the role of Serrifia marcescens as an organism causing severe and even fatal opportunisic infections in hospitalized patients, pigticularly in individuals whose host reswstance has been compromised by diseasefor therapeutic measures such as antibioutic or antimetabolite therapy. The fact that the majority of reports of this type infection have come from the USANis interesting, and whether this reflects a higher incidence in the occurrence $\mathrm{Nof}^{\circ}$ Serratia marcescens in that countrynor better methods of identification of the organism has been the subject of a previous communication. In the prefent study in a 600-bed Canadian teaching hospital, 114 strains of Serratia marcescens were isolated from 105 patients over the course of 10 months, 80 of the strains bing isolated in the first five months of 